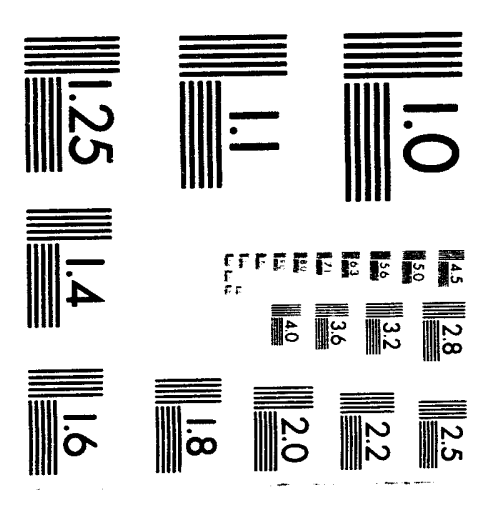



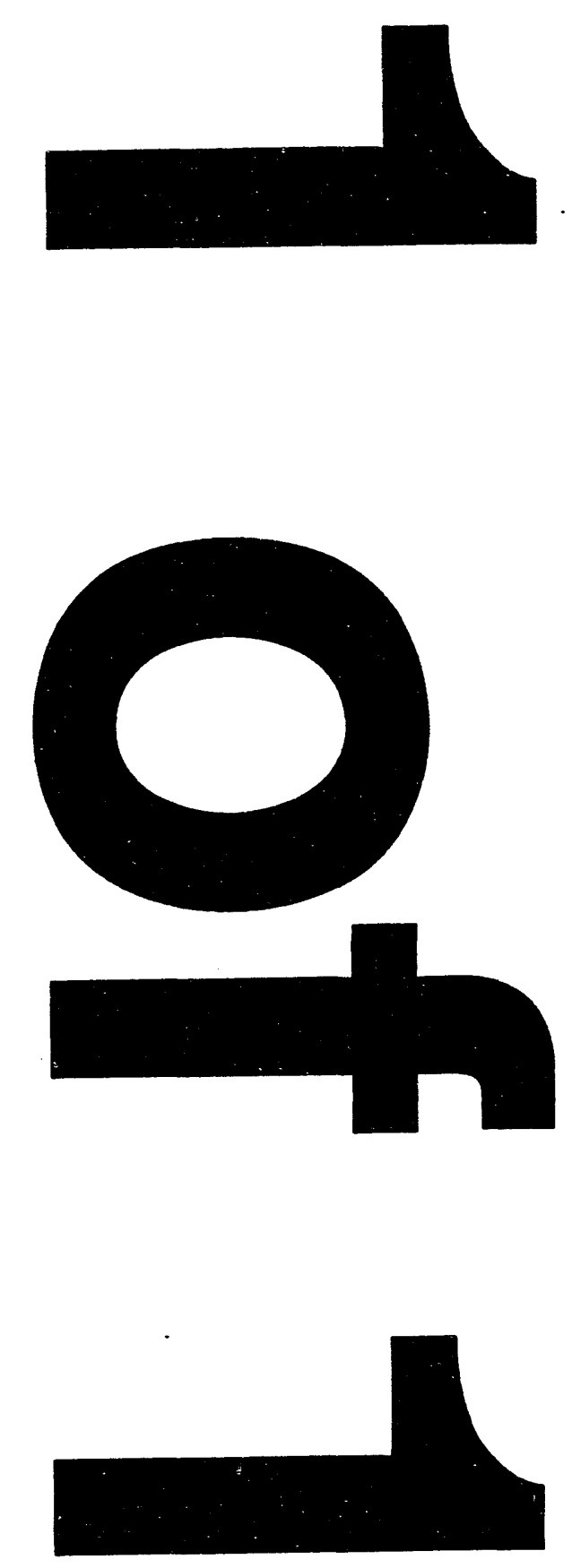
NÁTO Advanced Study. Institute on Hot and Dense Nuclear Matter, Bodrum, Turkey, September 26 - October 9, 1993.

$\mathrm{BNL}-49938$

\title{
COMPRESSION, EXPANSION, AND FREEZE-OUT IN NUCLEUS-NUCLEUS COLLISIONS AT THE AGS
}

\author{
Peter Braun-Munzinger \\ Department of Physics \\ SUNY at Stony Brook \\ Stony Brook, NY 11794-3800, USA

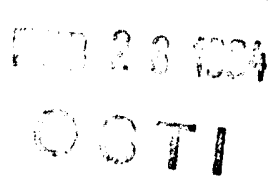

E814/E877 Collaboration:

J. Barrette ${ }^{3}$, R. Bellwied ${ }^{8}$, S. Bennett ${ }^{8}$, P. Braun-Munzinger ${ }^{6}$, W.E. Cleland ${ }^{5}$, T.M. Cormier $^{8}$, G. David ${ }^{6}$, J. Dee ${ }^{6}$, G.E. Diebold ${ }^{9}$, O. Dietzsch ${ }^{7}$, J.V. Germani ${ }^{9}$, S. Gilbert $^{3}$, S.V. Greene ${ }^{9}$, J.R. Hall ${ }^{8}$, T.K. Hemmick ${ }^{6}$, N. Herrmann ${ }^{2}$, B. Hong ${ }^{6}$, K. Jayananda ${ }^{5}$, D. Kraus ${ }^{5}$, B. Shiva Kumar ${ }^{9}$, R. Lacasse ${ }^{3}$, Q. Li ${ }^{8}$, A. Lukaszew ${ }^{8}$, W.J. Llope ${ }^{6}$, T.W. Ludlam $^{1}$, S. McCorkle 1 , R. Majka ${ }^{9}$, S.K. Mark ${ }^{3}$, R. Matheus ${ }^{8}$, J.T. Mitchell ${ }^{9}$, M. Muthuswamy $^{6}$, E. O'Brien ${ }^{1}$, S. Panitkin ${ }^{6}$, C. Pruneau ${ }^{8}$, M.N. Rao ${ }^{6}$, M. Rosati ${ }^{3}$, F. Rotondo $^{9}$, N.C. daSilva ${ }^{7}$ S. Sedykh ${ }^{6}$, U. Sonnadara ${ }^{5}$, J. Stachel ${ }^{6}$, H. Takai ${ }^{1}$, E.M. Takagui $^{7}$, J. Wessels ${ }^{6}$, C. Winter ${ }^{9}$, G. Wang ${ }^{3}$, D. Wolfe ${ }^{4}$, C.L. Woody ${ }^{1}$, N. Xu ${ }^{6}, Y$. Zhang $^{6}$, Z. Zhang ${ }^{5}$, C. Zou ${ }^{6}$

${ }^{1} \mathrm{BNL}-{ }^{2} \mathrm{GSI}-{ }^{3} \mathrm{McGill}$ Univ. $-{ }^{4}$ Univ. of New Mexico $-{ }^{5}$ Univ. of Pittsburgh - ${ }^{6} \mathrm{SUNY}$ Stony Brook $-{ }^{7}$ Univ. of São Paulo $-{ }^{8}$ Wayne State Univ. $-{ }^{9}$ Yale Univ.

\section{INTRODUCTION}

In the following we will present selected aspects of recent data on central nucleusnucleus collisions taken by the E814/E877 collaboration at the BNL-AGS. We will first demonstrate that stopping is nearly complete in these collisions, and discuss the initial energy and baryon densities inferred from model comparisons with the data. Next we will discuss the amount of expansion the system undergoes from the initial phase until freeze-out by presenting recent results on pion-pion correlations. The (baryonic) resonance composition of the system at freeze-out is obtained by analyzing pion spectra at low transverse momentum $p_{t}$ and by inspection of direct measurements of the $\Delta(1232)$ resonance. Based on analysis of these measurements we will then discuss a "local" freeze-out scenario, where the freeze-out volume is connected with the temperature averaged pion-nucleon cross section. A summary of the current results and of future plans for Au-Au collisions with the E877 apparatus concludes the paper.

The data presented were obtained for central $14.6 \mathrm{~A} \mathrm{GeV} / \mathrm{c} \mathrm{Si}+\mathrm{Al}$ and $\mathrm{Si}+\mathrm{Pb}$ collisions and 11.4 A GeV/c Au+Au collisions. The E814/E877 experimental setup has been described previously (see e.g. $[1,2]$ ); all data presented here were taken in runs in spring 1991 and 1992 in the 'open spectrometer configuration' described in [3]. The forward spectrometer covers in one fixed setting an angular range in the magnet bend plane of $-115<\theta_{x}<14 \mathrm{mr}$ and $-21<\theta_{y}<21 \mathrm{mr}$ perpendicular to it. Centrality is selected by measuring associated charged particle multiplicity and/or transverse energy.

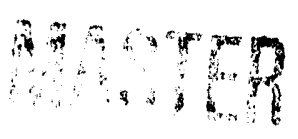


The degree of centrality selection is quantified by the ratio of the measured cross section $\sigma$ to the geometric cross section $\sigma_{g e o} \approx 3.6 \mathrm{~b}(1.6 \mathrm{~b})$ for $\mathrm{Si}+\mathrm{Pb}(\mathrm{Al})$ and $\approx 6 \mathrm{~b}$ for $\mathrm{Au}$ $+\mathrm{Au}$.

\section{BARYON RAPIDITY AND TRANSVERSE ENERGY DISTRIBUTIONS}

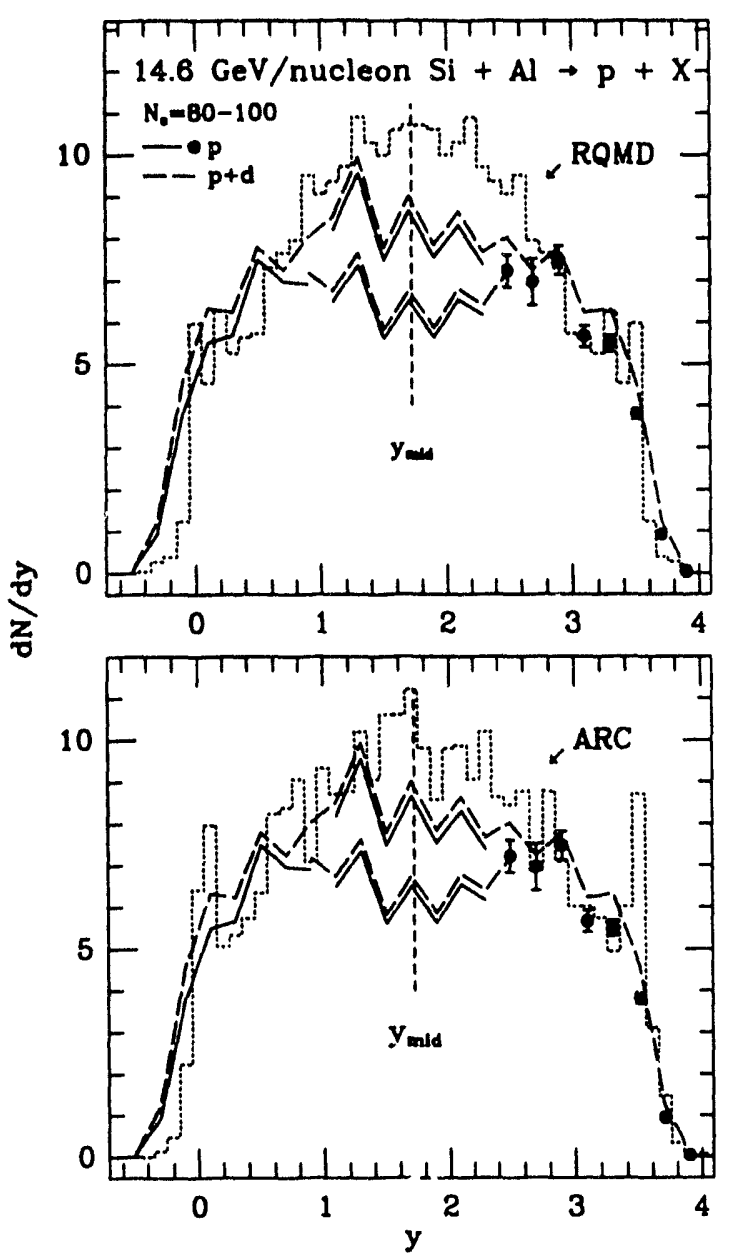

Figure 1: Proton rapidity distribution [3] (solid dots and lines) and sum of proton and deuteron distributions (long dashed lines) together with predictions from the event generators RQMD and ARC
The measured proton rapidity distribution for very central $\left(\sigma / \sigma_{\text {geo }}=0.2 \%\right) \mathrm{Si}$ $+\mathrm{Al}$ collisions is presented in Figure 1. The data are published in [3], where details about the setup, the analysis and the determination of systematic errors are described.

The absence of a peak at beam rapidity indicates that a large degree of stopping is achieved in the system. However, the distribution is much wider than expected for a fully stopped isotropic proton source where $\mathrm{dN} / \mathrm{dy} \propto \exp \left[-\left(\mathrm{y}-\mathrm{y}_{\mathrm{f}}\right)^{2} / 2 \cdot 0.34^{2}\right]$. A quantitative analysis of the degree of stopping for this system has been made in $[4,5]$. There it is shown that either the amount of stopping is about $65 \%$ or that the protons were initially fully stopped and expanded longitudinally with expansion velocity $\beta_{\|} \approx 0.6$.

To get the full baryon distribution we add, from a recent E814 analysis of deuteron distributions, the number of protons bound in deuterons to the proton distributions (see the dashed lines in Fig. 1). The data in Fig. 1 are compared to predictions from the event generators RQMD [6] and ARC [7], both based on cascading and resonance production. The overall degree of stopping is reasonably described by both models. The slight discrepancy at midrapidity could indicate that the (inverse) slope constant of the proton $m_{t}$ spectra is actually larger than we assumed for the evaluation of $\mathrm{dN} / \mathrm{dy}$.

Full stopping and very high baryon densities are also inferred from our recent measurements [2] of transverse energy production in $\mathrm{Au}+\mathrm{Au}$ collisions at $11.4 \mathrm{GeV} / \mathrm{c}$ per nucleon. Results for the angular distribution of the produced transverse energy are shown in Fig. 2. Values of $200 \mathrm{GeV} /$ unit of pseudorapidity $\eta$ are observed for the $\mathrm{Au}+\mathrm{Au}$ system, a factor of 8.7 larger than what is observed for $\mathrm{Si}+\mathrm{Al}$. Accounting for the different beam energy this implies a $48 \%$ increase in the central rapidity density of $E_{t}$ compared to what is expected from the available energy in the center-of-mass. In the ARC [7] and RQMD [6] models, which describe the transverse energy production rather well (see [2]), initial energy and baryon densities of $>1.5 \mathrm{GeV} / \mathrm{fm}^{3}$ and $>8 \mathrm{times}$ normal nuclear matter density are achieved over significant time periods $(>5 \mathrm{fm} / \mathrm{c}$ ) for these heavy systems. 


\section{PION CORRELATIONS AND SOURCE SIZE AT FREEZE-OUT}

The results of $\pi^{+} \pi^{+}$and $\pi^{-} \pi^{-}$correlation function measurements for cen$\operatorname{tral~} \mathrm{Si}+\mathrm{Pb}$ collisions (top $10 \%$ ) [8] are presented in Figure 3. Because of the correlation between momentum and space for pions and its strong influence on the correlation function measured within a finite acceptance we do not attempt to extract source radii directly from a fit to the data. Rather we compare our measured correlation functions with predictions from the event generator RQMD [6], using the Koonin/Pratt formalism to compute the correlation function within our spectrometer acceptance. For more details see [8].

Fig. 3 shows this comparison. Excellent agreement is found for both $\pi^{+} \pi^{+}$and $\pi^{-} \pi^{-}$correlation functions. The shape of the RQMD source for pions at freeze-out, defined by the distribution of space-time points corresponding to the last (strong) interaction of the pions, was determined from events corresponding to roughly the

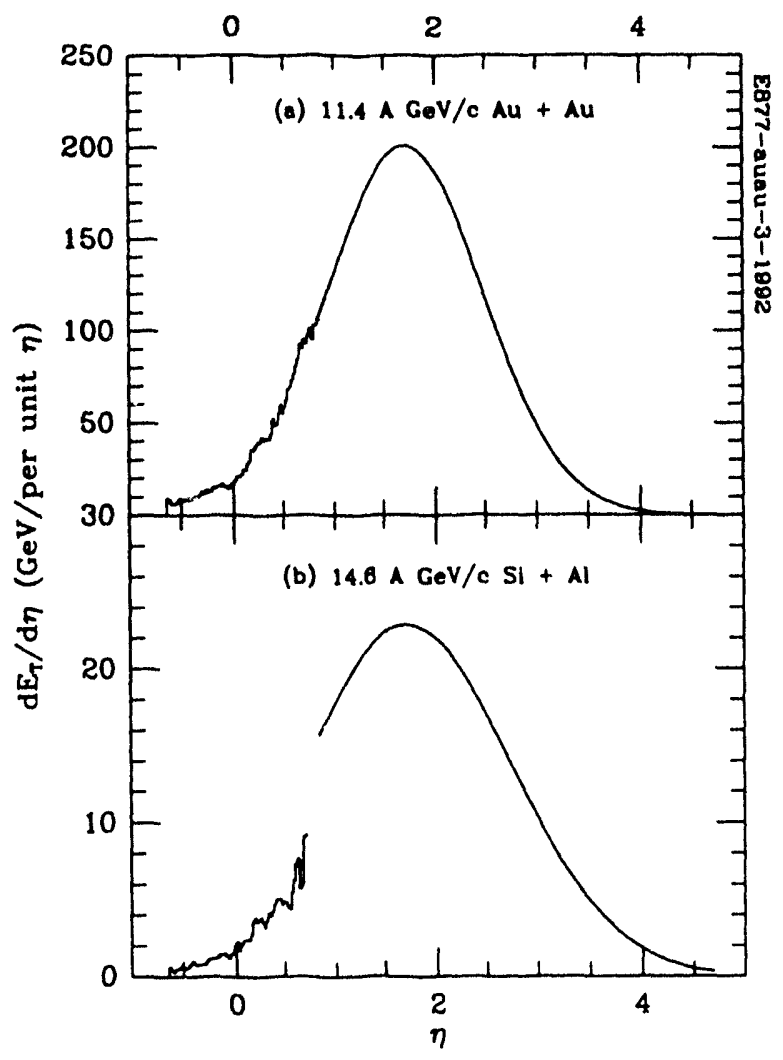

Figure 2: Experimental $d E_{t} / d \eta$ distributions for central $\mathrm{Si}+\mathrm{Al}$ and $\mathrm{Au}+\mathrm{Au}$ collisions same centrality as in the data. This analysis yielded a nearly spuerical source size of transverse radius $R_{T}=6.7 \mathrm{fm}$ and longitudinal radius (in the c.m. frame) $R_{L}=5.0$ $\mathrm{fm}$, resulting in a rms radius of $\mathrm{R}=8.3 \mathrm{fm}$. The agreement between data and RQMD calculations shown in Fig. 3 implies that our measured correlations are consistent with such a large source.

The rms radius of the source should be compared to the rms radius of $\mathrm{Si}$ of $3.04 \mathrm{fm}$ [9]. This corresponds to a transverse radius of $R_{T}(\mathrm{Si})=2.1 \mathrm{fm}$ yielding a transverse expansion of the sytem of more than a factor of 3 . Because of the resonance decays (see below) it is difficult to get a unique result on transverse expansion velocities. Preliminary results [5] indicate $\beta_{\perp} \approx 0.5$, leading to an estimate for the collective expansion time of about $10 \mathrm{fm} / \mathrm{c}$. We also note that very similar (large) source radii are obtained from recent E814 measurements of the deuteron to proton ratio in central $\mathrm{Si}+\mathrm{Pb}$ collisions and its interpretation in a thermal model [10].

\section{PARTICLE SPECTRA AND RESONANCE MATTER}

The shape of pion spectra as funciion of transverse momenturn or transverse mass contains information about the collision dynamics. For example, pion emission from an equilibrized system with temperature $T_{B}$ leads to a Boltzmann distribution for the invariant cross section, i.e. $\sigma_{i n v} \propto m_{t} \cdot \exp \left(-m_{t} / T_{B}\right)$, with the transverse mass $m_{t}=\sqrt{m^{2}+p_{t}^{2}}$. The Boltzmann shape can be modified, particularly at low values of transverse momentum, by pions resulting from the decay of resonances at freeze-out (see below for a definition of freeze-out), even if the system stays in thermal equilibrium throughout [11]. Analysis of this low $p_{t}$ component then yields information about the population of, in particular, nucleon excited states.

Figure 4 shows the $\pi^{-}$spectrum plotted versus transverse mass $m_{t}$ divided by a 
Boltzmann spectrum $\sigma_{\text {inv }} \propto m_{t} \cdot \exp \left(-m_{t} / T_{B}\right)$ fitted to the range $p_{t} \geq 0.3 \mathrm{GeV} / \mathrm{c}$. The data are for central (top $2 \%$ ) Si $+\mathrm{Pb}$ collisions. More details and results for other systems and pion charges can be found in [12]. For $m_{t}-m_{\pi}$ values larger than 0.2 all spectra very closely follow a thermal shape. At smaller transverse mass, they exhibit a significant enhancement over a thermal distribution. We find very similar enhancement in both $\pi^{+}$and $\pi^{-}$spectra, and for $\mathrm{Si}+\mathrm{Al}$ collisions.

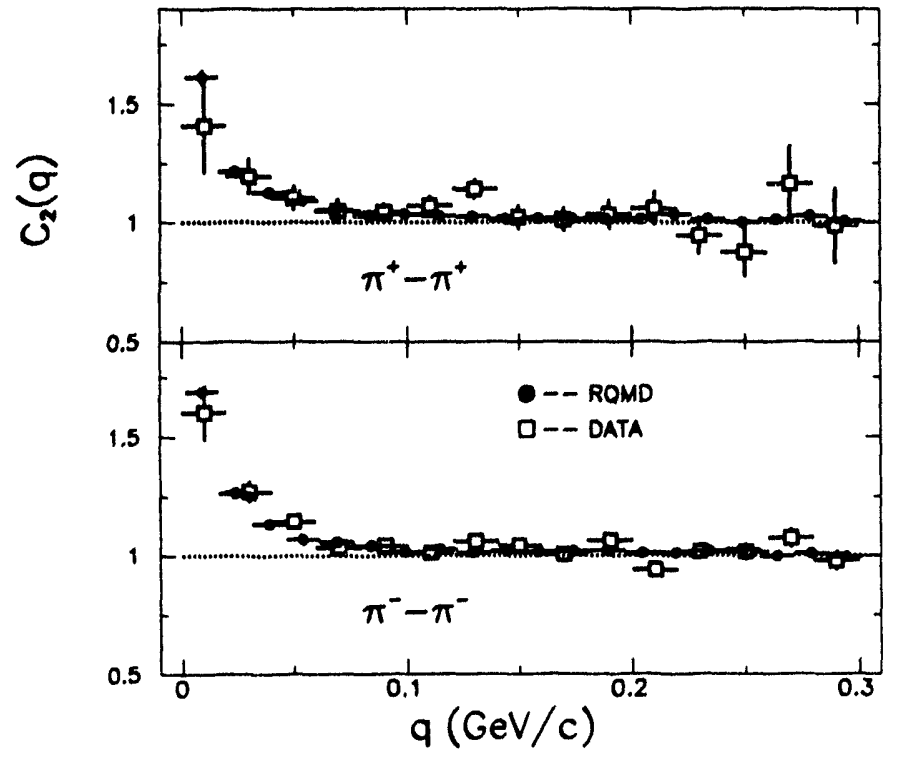

Figure 3: Experimental 2 pion correlation function together with the corresponding correlation function constructed from RQMD events (see [8])
To show the sensitivity of the transverse momentum spectra to the resonance content at freezeout, we also plot, in Fig. 4, the predictions for the spectral shape assuming various amounts of $\Delta(1232)$ resonance excitation. Assuming a ratio of pions from $\Delta$ decay to direct (thermal) pions of $\pi_{\Delta} / \pi_{T}$ between 0.4 and 0.6 nicely brackets all the data.

From the number of observed pions and nucleons (see, e.g., [13, $3,12])$ we get $(\pi / N)_{\text {exp }}=\left(\pi_{\Delta}+\right.$ $\left.\pi_{T}\right) /(N+\Delta) \approx 1.1$. This then implies that

$$
\begin{aligned}
& \frac{\Delta}{\Delta+N}=\frac{\pi_{\text {Delta }}}{\pi_{D_{\text {elita a }}+\pi_{T}}} \frac{\pi_{\text {Delta }}+\pi_{T}}{\Delta+N}= \\
& 0.37 \pm 0.05 . \text { As discussed in [12], } \\
& \text { RQMD reproduces our pion spec- }
\end{aligned}
$$

tra extremely well and there the rapidity averaged fraction of nucleons in the $\Delta(1232)$ resonance at freezeout is $35 \%$ [14], in good agreement with the above number.

In Figure 5 it is shown that the $\Delta(1232)$ resonance is also seen directly in E814 by reconstructing the $\mathrm{p} \pi^{+}$invariant mass in central (top $2 \%$ ) $\mathrm{Si}+\mathrm{Pb}$ collisions. From the observed number of $\Delta$ resonance states one can determine their multiplicity. Taking into account the acceptance of the E814 spectrometer for $\Delta^{++}$reconstruction of $1.5 \cdot 10^{-3}$ for the rapidity interval $\mathrm{y}=1.9-3.1$ leads to a $\Delta^{++}$multiplicity of $2.1 \pm 0.7$. For comparison, we measured [3] 5.9 protons in the same rapidity interval. RQMD predicts $1.8 \Delta^{++}$for this interval in good agreement with our preliminary experimental number.

The measured $\Delta$ resonance population can also be understood in the context of a purely thermal model. To illustrate this, and to determine the temperature $\mathrm{T}$ of the equilibrized system from the measured $\Delta$ to nucleon ratio we present in Fig. 6 as a function of the temperature $T$, the population probabilities of all non-strange nucleon resonances with masses less than $2 \mathrm{GeV}$. In this calculation the widths of all states and, of course, all statistical factors have been included.

Clearly the $\Delta$ resonance is the dominant excited state. However, because of the many heavier resonances, the population of the $\Delta$ never exceeds about $45 \%$. From our measured $\Delta$ to nucleon ratio of $0.37 \pm 0.05$ we conclude that $T=0.14 \pm 0.02 \mathrm{GeV}$. This is consistent with the temperature determination from the analysis of pion spectra, where $T \approx 0.15 \mathrm{GeV}[4]$.

While the enhancement at low $p_{t}$ in pion spectra is well understood in terms of baryon resonance decays, recent preliminary E814 data imply [4] that there is also a strong enhancement at low $p_{t}$ in kaon spectra. The source of this enhancement is presently not understood: resonance decays are too weak to explain the size of the 
effect. An exciting possibility is that the kaon enhancement is due to a drop of the kaon mass in the hot and dense medium formed during the collision. Data recently taken with $\mathrm{Au}$ beams should shed light on this situation.

\section{FREEZE-OUT SCENARIO}

At freeze-out we assume that the system consists of a gas of pions and nucleons. The dominant cross section is then the pion-nucleon cross section $\sigma_{\pi N}$. We define freeze-out of the expanding system by requiring that at freeze out $\left\langle d_{\pi N}\right\rangle=$ $\sqrt{\left\langle\sigma_{\pi N}\right\rangle / \pi}$. Here, $\left\langle d_{\pi N}\right\rangle$ is the mean distance of a pion and the nearest nucleon in the gas, and $\left\langle\sigma_{\pi N}\right\rangle$ is the pion-nucleon cross section averaged over spin, isospin, and the Boltzmann distribution of pions and nucleons at the freeze-out temperature $\mathrm{T}_{f}$. From the previous arguments about spectral shapes and resonance population we deduce $\mathrm{T}_{f} \approx 0.14 \mathrm{GeV}$. At this temperature, $\left\langle\sigma_{\pi N}\right\rangle \approx 70 \mathrm{mb}$. Using a simple Monte Carlo procedure we have determined that $<d_{\pi N}>\approx \frac{0.75}{\left(n_{\pi}+n_{N}\right)^{1 / 3}}$, where $n_{\pi}$ and $n_{N}$ are the pion and nucleon number densities at freeze-out. Note that this implies that the freeze-out radius $\mathrm{R}_{f} \propto$ $d N / d y^{1 / 3}$. For the freeze-out volume $V_{f}$ we obtain:

$$
V_{f}=\left(\frac{\left\langle\sigma_{\pi N}>\right.}{\pi}\right)^{3 / 2} \frac{N_{\pi}+N_{N}}{0.75^{3}}
$$

Here, $\mathrm{N}_{\pi}$ and $\mathrm{N}_{N}$ are the number of pions and nucleons in the fireball. A freeze-out volume directly proportional to the total particle multiplicity, i.e. freeze-out at constant density, was already discussed long ago by Pomeranchuk [15].

For the system $\mathrm{Si}+\mathrm{Pb}$ and very central collisions we obtain $[13,3,12] \mathrm{N}_{N} \approx 138$ and $N_{\pi} \approx 150$, depending somewhat on what rapidity range is included. This im-

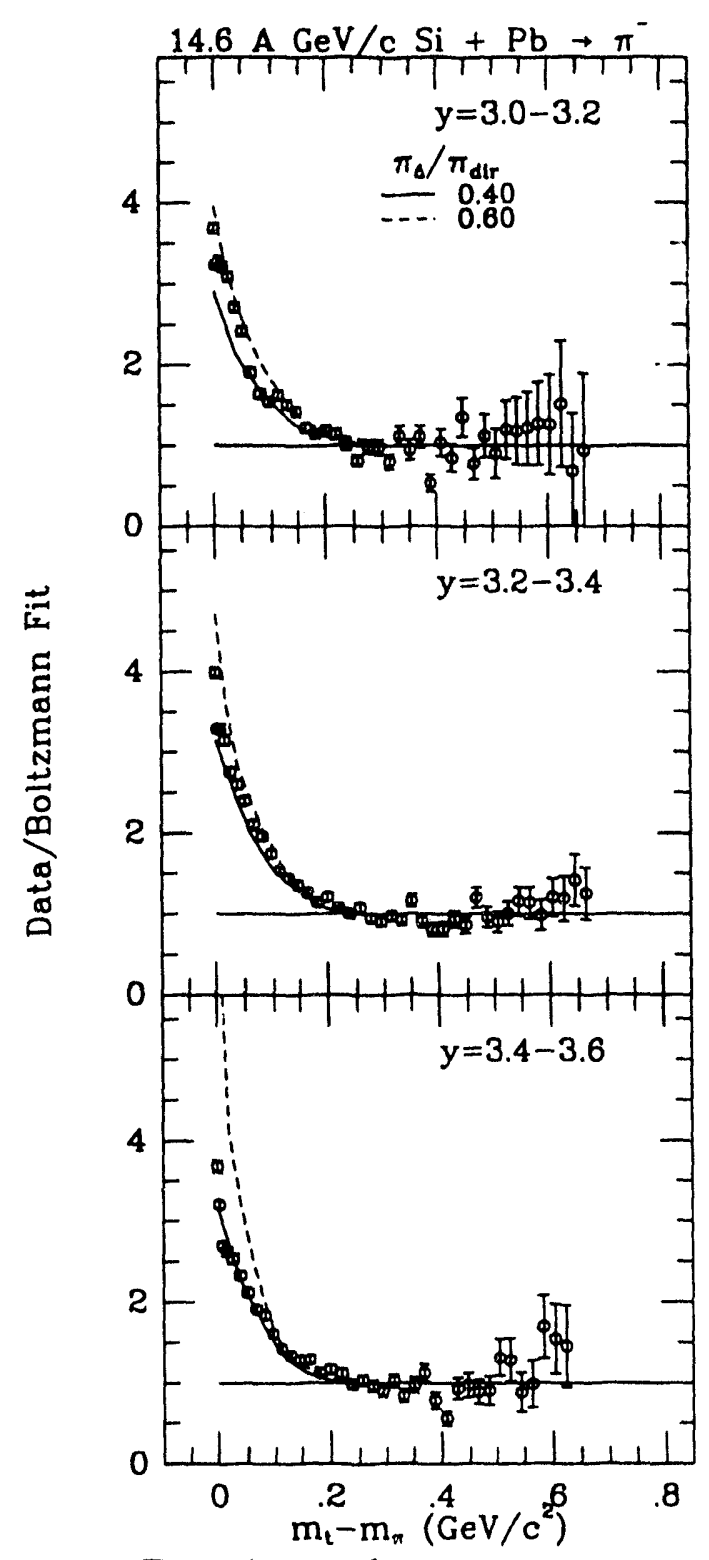

Figure 4: Experimental $\pi^{-}$spectra normalized to a Boltzmann distribution fitted to the data for $p_{t} \geq 0.3 \mathrm{GeV} / \mathrm{c}$. Solid and dashed lines: thermal model with different fractions of $\Delta$ decay vs. direct pions. plies a freeze-out volume of $V_{f}=2270 \mathrm{fm}^{3}$ corresponding to $R_{f}=8.1 \mathrm{fm}$. The equivalent rms radius is then $6.3 \mathrm{fm}$, somewhat smaller than that determined from the correlation analysis as discussed above. The resulting pion and nucleon densities at freeze-out are $n_{\pi}=0.066 / \mathrm{fm}^{3}$ and $n_{N}=0.061 / \mathrm{fm}^{3}$. From $\mathrm{T}_{f}$ and the particle densities $n_{N}$ and $n_{\pi}$ we can determine both the pion and nucleon chemical potentials via

$$
n=\frac{g}{2 \pi^{2}} \int \frac{p^{2} d p}{\exp \left((E-\mu) / T_{f}\right) \pm 1}
$$

with $g=4$ for nucleons and $g=1$ for each pion charge. A (small) excluded volume correction [16] is also applied. This yields for the nucleon chemical potential $\mu_{B}=$ 
$0.51 \mathrm{GeV}$. For the pion chemical potential we get $\left|\mu_{\pi}\right|<30 \mathrm{MeV}$, consistent with zero and not in agreement with the large values used recently [17] to explain the low $p_{t}$ enhancement in pion spectra. From the measured $K^{+} / K^{-}$ratio [18] one carl also determine the strangeness chemical potential $\mu_{S}=0.105 \mathrm{GeV}$.

If the system is in equilibrium at freeze-out, the baryon and strangeness chemical potentials determine uniquely the baryon production ratios $\bar{p} / p=7 \cdot 10^{-4}$ and $\bar{\Lambda} / \Lambda=$ $3.7 \cdot 10^{-3}$. Both ratios are close to what is observed experimentally, namely $(\bar{p} / p)_{\exp }=$ $(6 \pm 3) \cdot 10^{-4}[19]$, and $(\bar{\Lambda} / \Lambda)_{\exp }=(2 \pm 1) \cdot 10^{-3}[20]$, lending further support to the equilibrium scenario.

In addition to the chemical potentials, the particle densities and freeze-out temperature also completely determine the total entropy of the system via [15]

$$
S_{f}=\frac{V_{f}}{T_{f}} \sum_{i=\pi, N} g_{i} \int \frac{p^{2} d p}{2 \pi^{2}} \frac{p^{2} / 3 E_{i}-\mu_{i}+E_{i}}{\exp \frac{E_{i}-\mu_{i}}{T_{f}} \pm 1}
$$

Numerical evaluation leads to an entropy per baryon of $S_{f} / N_{N}=15$ for $T_{f}=0.14$ $\mathrm{GeV}$. Alternatively, one can also, from the measured ratios [10] of deuterons/protons and pions/baryons deduce the entropy/baryon of the system, using the Siemens and Kapusta formula [21], with a result of $S_{f} / N_{N}=13$, in beautiful agreement with the entropy from the freeze-out scenario.

\section{SUMMARY AND FUTURE PLANS}

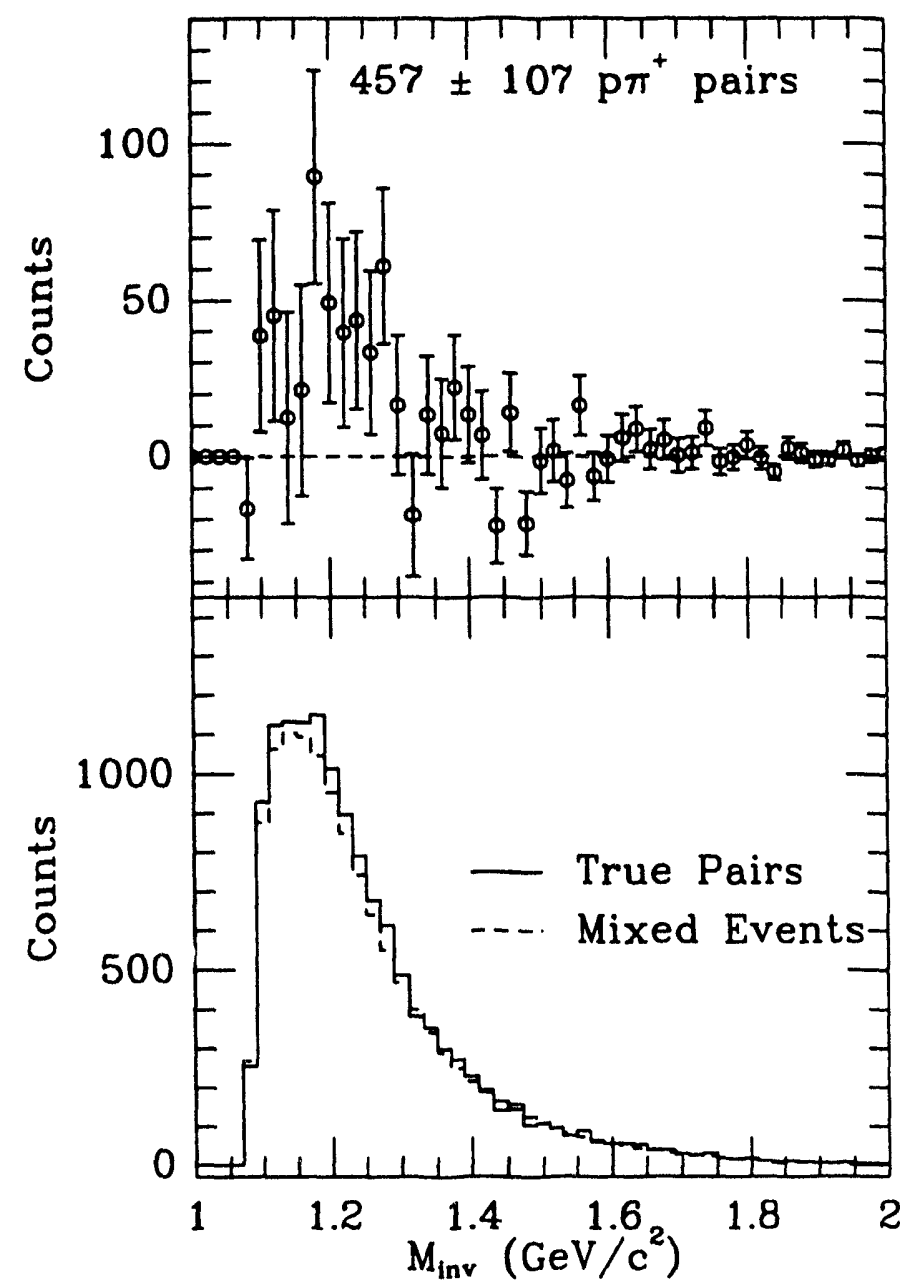

Figure 5: Reconstruction of the $\Delta^{++}$resonance for central $\mathrm{Si}-\mathrm{Pb}$ collisions.
We have demonstrated, using recent data from the E814/E877 collaboration and from the AGS heavy ion program in general, that strong evidence exists for the formation of a hot and dense fireball at AGS energies. Comparison to models successfully describing our data implies that $\epsilon \approx 1.5 \mathrm{GeV} / \mathrm{fm}^{3}$ and $\rho / \rho_{0} \approx$ 8 are achieved for significant timescales $(\approx 5 \mathrm{fm} / \mathrm{c})$, especially for the $\mathrm{Au}+\mathrm{Au}$ system. A considerable transverse expansion (by about a factor three) ensues, leading to a freeze-out radius of $8.3 \mathrm{fm}(\mathrm{rms})$ for $\mathrm{Si}+\mathrm{Pb}$. Analysis of the $\Delta$ resonance population shows that about $37 \%$ of the baryons are excited to the $\Delta$ resonance. This and the inverse slope constants of pion $m_{t}$ spectra imply a freeze-out temperature of $\mathrm{T}_{f} \approx 0.14 \mathrm{GeV}$. This leads to a consistent freeze-out scenario, from which the pion and baryon chemical potentials and the entropy at freeze-out are determined.

The future plans of our collaboration (E877) are centered on the analysis of the recent run with $\mathrm{Au}$ projectiles at $11 \mathrm{GeV} /$ nucleon. These data will be analyzed to provide, e.g., threedimensional information on pion source sizes, a high statistics measurement of the low 


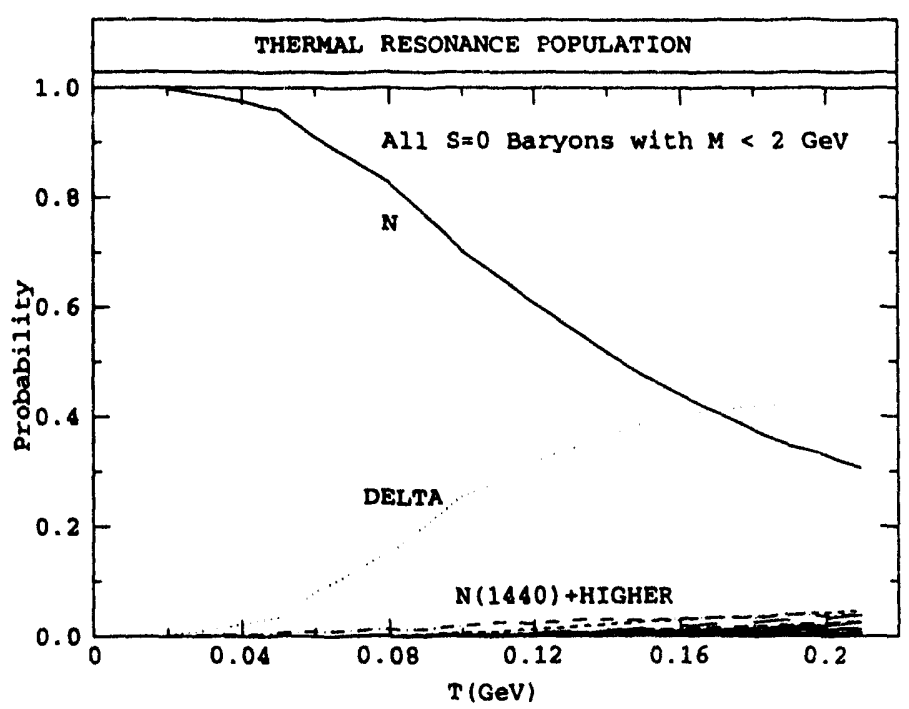

Figure 6: Population of Nucleon Resonances as function of the temperature. The widths of the resonances is included in the calculation.

$p_{t}$ part of the kaon transverse momentum spectra to hopefully confirm our intriguing $\mathrm{Si}+\mathrm{Pb}$ results, and a first look at inclusive photon spectra following central $\mathrm{Au}+\mathrm{Au}$ collisions. In addition, preliminary analysis of transverse energy distributions has yielded a first indication of collective transverse flow and this direction will also be vigorously pursued.

Financial support by the US DoE, the NSF, the Canadian NSERC, and CNPq Brazil is gratefully acknowledged.

\section{References}

[1] J. Barrette et al., E814 Coll., Phys. Rev. Lett. 64 (1990) 1219; Phys. Rev. C45 (1992) 819; Phys. Rev. C46 (1992) 312.

[2] J. Barrette et al., E814/E877 Coll., Phys. Rev. Lett. 70 (1993) 2996.

[3] J. Barrette et al., E814 Coll., Z. Physik C59 (1993) 211.

[4] J. Stachel, Invited Paper, Quark Matter '93, Borlänge, Sweden, June 1993, Nucl. Phys. A (in print)

[5] P. Braun-Munzinger, J. Stachel, N. Xu, unpublished

[6] H. Sorge, H. Stöcker, W. Greiner, Ann. Phys. (NY) 192 (1989) 266; A. Jahns, H. Sorge, H. Stöcker, W. Greiner, Z. Physik A341 (1992) 243.

[7] T. J. Schlagel, S. H. Kahana and Y. Pang, Phys. Rev. Lett. 69 (1992) 3290.

[8] N. Xu, Contributed Paper Quark Matter '93, Borlänge, Sweden, June 1993, Nucl. Phys. A (in print)

[9] H. Überall, Electron Scattering from Complex Nuclei, Academic Press, N.Y. 1971, p. 210.

[10] J. Germani, Ph.D. thesis, Yale Univ., July 1993, and J. Barrette et al., E814 Coll., preprint Dec. 1993

[11] G.E. Brown, J. Stachel, and G.M. Welke, Phys. Lett. B253 (1991) 19. 
[12] T.K. Hemmick, Contributed Paper, Quark Matter '93, Borlänge, Sweden, June 1993, Nucl. Phys. A (in print)

[13] C. Parsons, proceedings Workshop HIPAGS '93, MITLNS-2158.

[14] M. Hofmann and R. Mattiello, private communication.

[15] E. Shuryak, "The QCD Vacuum, Hadrons and the Superdense Matter", World Scientific, Singapore 1988, p. 333

[16] J. Cleymans and H. Satz, Z. Physik C57(1993)135

[17] J.L. Goity and M. Leutwyler, Phys. Lett 228B(1989)517; M. Kataja and V. Ruuskanen, ibid 243B(1990)181

[18] T. Sung, proceedings Workshop HIPAGS '93, MITLNS-2158.

[19] see e.g. overview by H.J. Crawford, proceedings Workshop HIPAGS '93, MITLNS2158 and refs. there.

[20] G.S.F. Stephans, Invited Paper, Quark Matter '93, Borlänge, Sweden, June 1993, Nucl. Phys. A (in print)

[21] P. Siemens and J. Kapusta, Phys. Rev. Lett. 43(1978)1486, 1690E 

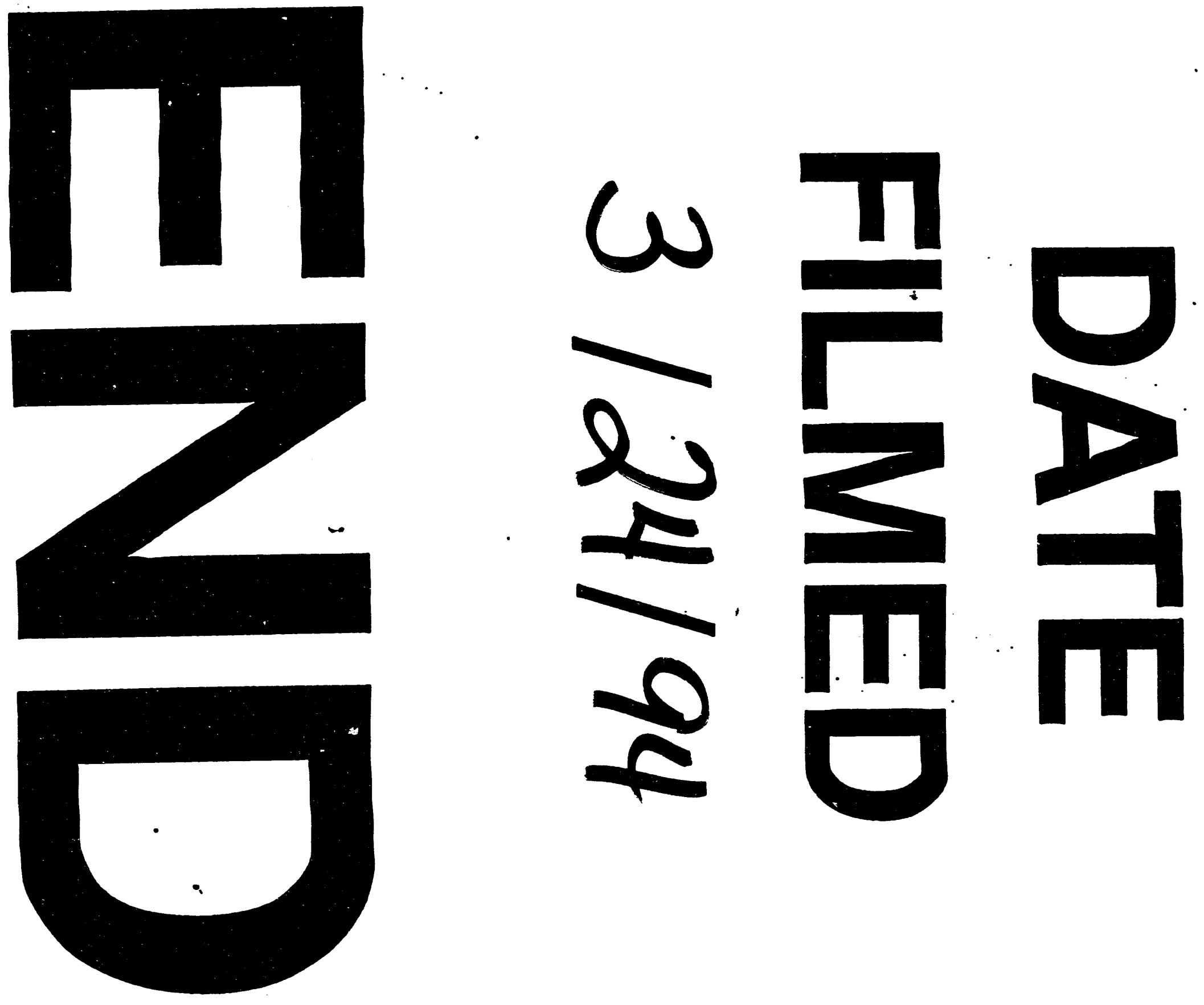

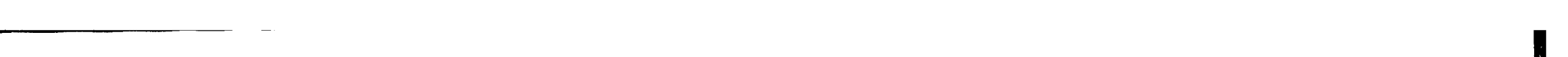